\title{
Desinserção do tronco braquiocefálico em traumatismo do tórax
}

\author{
José Carlos R. IGLÉZIAS*. Divino Francisco PINTO*, Luiz Felipe P. MOREIRA*, Luís Alberto DALLAN*, \\ Geraldo VERGINELLI*, Adib D. JATENE*
}

RBCCV 44205-161

\begin{abstract}
IGLÉZIAS, J. C. R.; PINTO, D. F.; MOREIRA, L. F. P.; DALLAN, L. A.; VERGINELLI, G.; JATENE, A. D. Desinserção do tronco branquiocefálico em traumatismo fechado do tórax. Rev. Bras. Cir. Cardiovasc., 7(1):44-47, 1992.

RESUMO: É relatado o caso de paciente adulto, de 48 anos de idade, politraumatizado, vítima de acidente automobilístico. O mesmo deu entrada no serviço de emergência do InCór em choque, com múltiplas fraturas de arcos costais, pneumotórax total à direita, fratura de fêmur e alargamento importante do mediastino aos raios $\mathrm{X}$. Foi compensado hemodinamicamente, monitorizado e submetido a angiocardiografia, que evidenciou rotura de aorta ao nível do tronco branquiocefálico, tratado cirurgicamente com interposiçāo de um tubo de Dacron entre a aorta ascendente e o tronco braquiocefálico. Dá-se ênfase ao modo como o caso foi conduzido, o que permitiu o êxito do tratamento. Inicialmente, a circulação extracorpórea foi estabelecida entre os vasos femorais e, só após fibrilação ventricular, o tórax foi aberto. Isto determinou o destamponamento da lesão aórtica, permitiu a sutura do local da desinserção do tronco braquiocefálico na aorta e a interposição de um enxerto de Dracon entre os dois vasos, restabelecendo, plenamente, o fluxo cerebral.
\end{abstract}

DESCRITORES: tronco braquiocefálico, cirurgia; traumatismos do tórax.

\section{INTRODUÇÃO}

A rotura da aorta é lesão traumática do tórax que mais comumente compromete o coração ou os grandes vasos da base. O primeiro caso de rotura da aorta devido a trauma fechado foi relatado por Vesalius, em 1557, (citado por SYMBAS ${ }^{\circ}$ ) e sua freqüência relativa pode ser extrapolada pelo dado de que uma em cada seis vítimas de acidente automobilístico que morre por trauma fechado sobre o tórax, apresenta rotura de aorta ${ }^{2}$. Em menor grau, a rotura aórtica também ocorre por quedas de grandes altitudes, ou outros tipos de lesões por esmagamento.

A rotura ocorre no ístmo em $90 \%$ dos casos e dilacerações múltiplas podem estar presentes em alguns pacientes; em outros, as extremidades da aorta rota podem estar separadas por vários centímetros, produzindo hematoma mediastinal, ou um pseudo-aneurisma.

Calcula-se que $10 \%$ a $20 \%$ dos pacientes com rotura da aorta vivem o suficiente para serem tratados com êxito em circunstâncias ideais ${ }^{5}$, o que inclui alto nível de percepção da possibilidade de rotura aórtica em vítimas de acidente automobilístico, bem como a abordagem por equipe bem coordenada '. Do mesmo modo que na lesão cardíaca, a rotura da aorta pode estar mascarada por lesões de outros órgãos e o diagnóstico pode passar desapercebido. Os pacientes muitas vezes se queixam de dores nas costas, além da dor torácica, de modo análogo aos pacientes com dissecção aórtica. Se o hematoma mediastinal em expansão, ou o pseudo-aneurisma, estreita o lúmen aórtico, ou se a média e a íntima, dilaceradas, levarem a obstrução aórtica parcial, pode resultar isquemia da medula espinal e dos rins.

A tríade disgnóstica que ocorre em mais da metade dos casos de rotura da aorta consiste em: 1 ) aumento da pressão arterial e da amplitude do pulso nas extremidades superiores; 2) diminuição da pressão e da amplitude do pulso nas extremidades infe-

Trabalho realizado no Instituto do Coração do Hospital das Clínicas da Faculdade de Medicina da Universidade de São Paulo. São Paulo, SP, Brasil. Recebido para publicação em 3 de janeiro de 1992.

* Do Instituto do Coração do Hospital das Clínicas da Faculdade de Medicina da Universidade de São Paulo.

Endereço para separatas: José Carlos Iglézias. Av. Dr. Enéas de Carvalho Aguiar, 44. Divisão Cirúrgica. 05403 São Paulo, SP, Brasil. 
IGLÉZIAS, J. C. R.; PINTO, D. F.; MOREIRA, L. F. P.; DALLAN, L. A.; VERGINELLI, G.; JATENE, A. D. - Desinserção do tronco branquiocefálico em traumatismo fechado do tórax. Rev. Bras. Cir. Cardiovasc., 7(1):44-47, 1992.

riores; 3) evidências radiológicas de alargamento do mediastino superior ${ }^{7.4}$.

O objetivo deste trabalho é o de ressaltar que o paciente politraumatizado, quando atendido em local que reúna equipe habilitada para o diagnóstico, pode, quando bem conduzido, levar ao exito o tratamento da lesão cardíaca e/ou dos grandes vasos.

\section{RELATO DO CASO}

Paciente do sexo masculino, 48 anos de idade, vítima de acidente automobilístico em 24/01/90, deu entrada no PS do InCór poucas horas após, em estado de choque. Foram constatados: afundamento torácico, com respiração paradoxal, fratura de ossos da perna. Foi compensado hemodinamicamente e
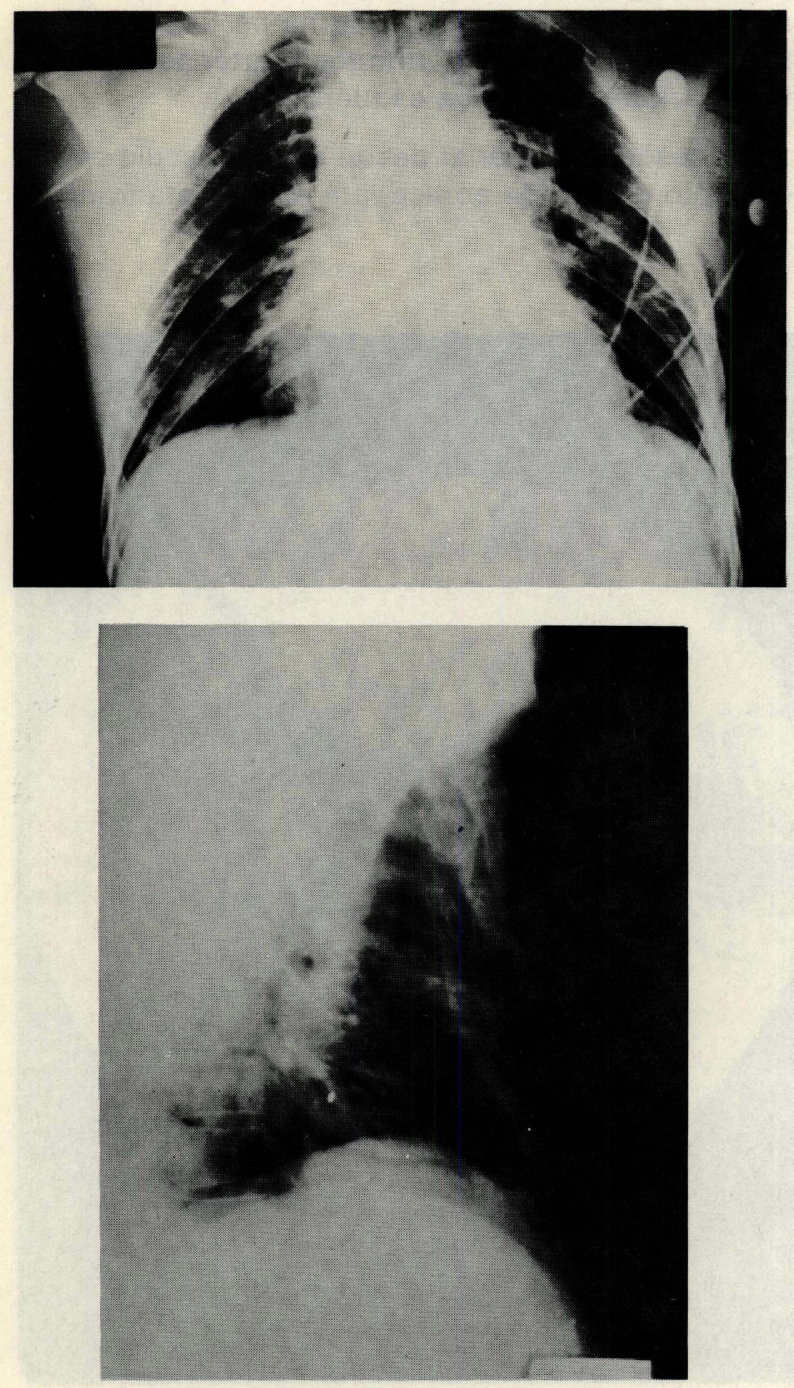

Fig. 1 - Radiografia de tórax pré-operatória.

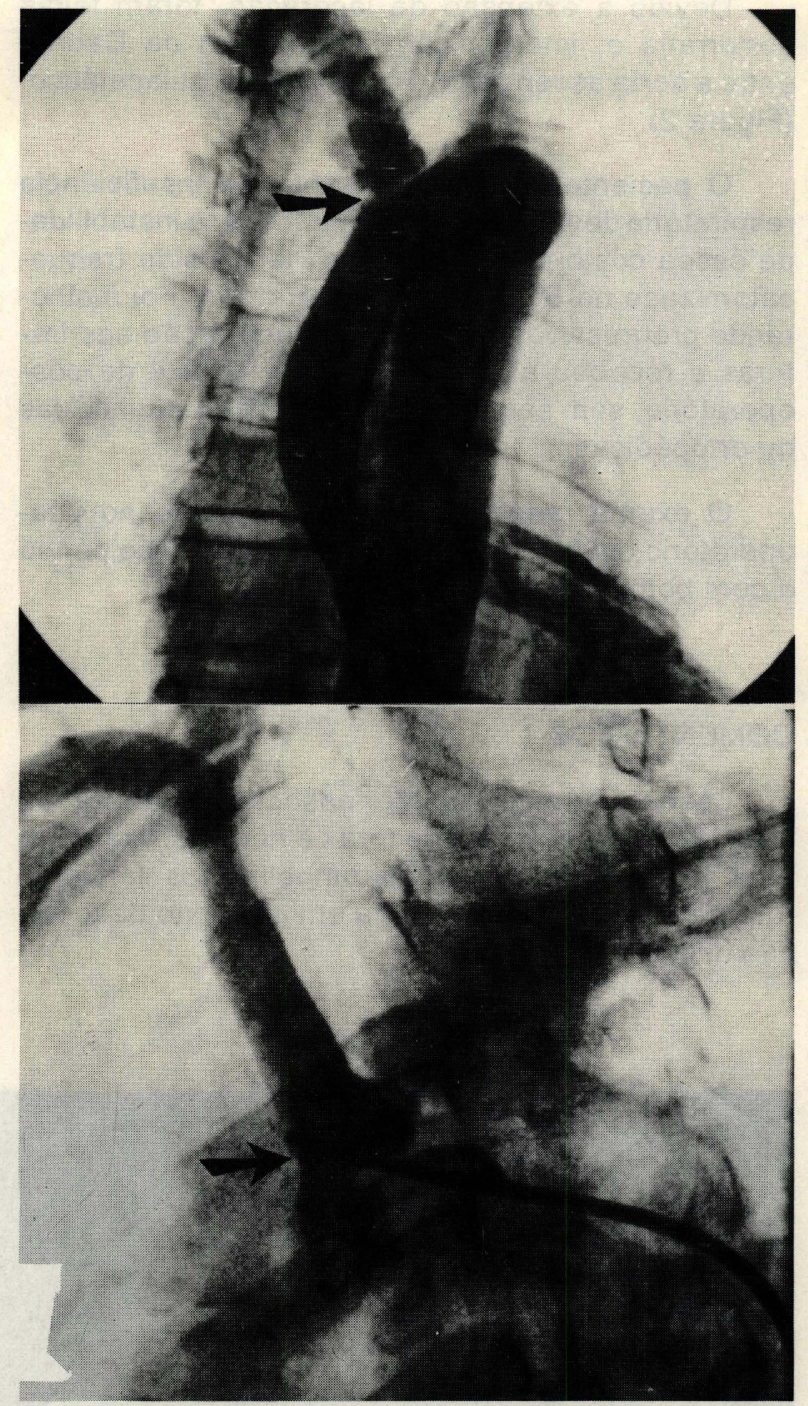

Fig. 2 - Aortografia no pré-operatório.

submetido a radiografia de tórax, que evidenciou pneumotórax total à esquerda, fratura de esterno e alargamento do mediastino. Após a drenagem de cavidade pleural esquerda, foi submetido a angiocardiografia (Figura 1), que permitiu o diagnóstico de rotura de aorta ascendente ao nível do tronco branquiocefálico e tamponamento cardíaco.

Foi submetido a circulação extracorpórea através dos vasos femorais, até o momento em que o coração apresentou fibrilação ventricular devido ao resfriamento; realizada a toracotomia, que determinou o destamponamento da ferida operatória e permitiu a compressão digital da lesão. Havia rotura da aorta ao nível do tronco branquiocefálico, o qual permaneceu unido à aorta somente através de sua adventícia e, nesta última, encontramos esgarçamento, através do qual o sangue fluía para o mediastino. 
IGLÉZIAS, J. C. R.; PINTO, D. F.; MOREIRA, L. F. P.; DALLAN, L. A.; VERGINELLI, G.; JATENE, A. D. - Desinserçäo do tronco branquiocefálico em traumatismo fechado do tórax. Rev. Bras. Cir. Cardiovasc., 7(1):44-47, 1992.

Devido à extensão da laceração, foram feitas aortorrafia e, interposição de um tubo de Dacron entre a aorta ascendente e o tronco branquiocefálico. (Figura 2).

O paciente evoluiu apresentando insuficiência respiratória devido a contusão pulmonar e instabilidade óssea costo-condro-esternal, tendo sido traqueostomizado no $5^{\circ}$ dia de pós-operatório. Foi melhorando progressivamente, com estabilização das fraturas e recebeu alta hospitalar no $22^{\circ}$ dia de pósoperatório, sem seqüelas neurológicas, circulatórias ou ortopédicas.

O exame radiológico digital realizado no pósoperatório evidenciou tronco branquiocefálico pérvio e com bom fluxo (Figura 3 ).

\section{COMENTÁRIOS}

Lesões aórticas estão associadas a graves traumatismos fechados do tórax e não são raras. Em uma série de acidentes automobilísticos fatais, a rotura da aorta foi encontrada em um sexto de todas as vítimas ${ }^{2}$.
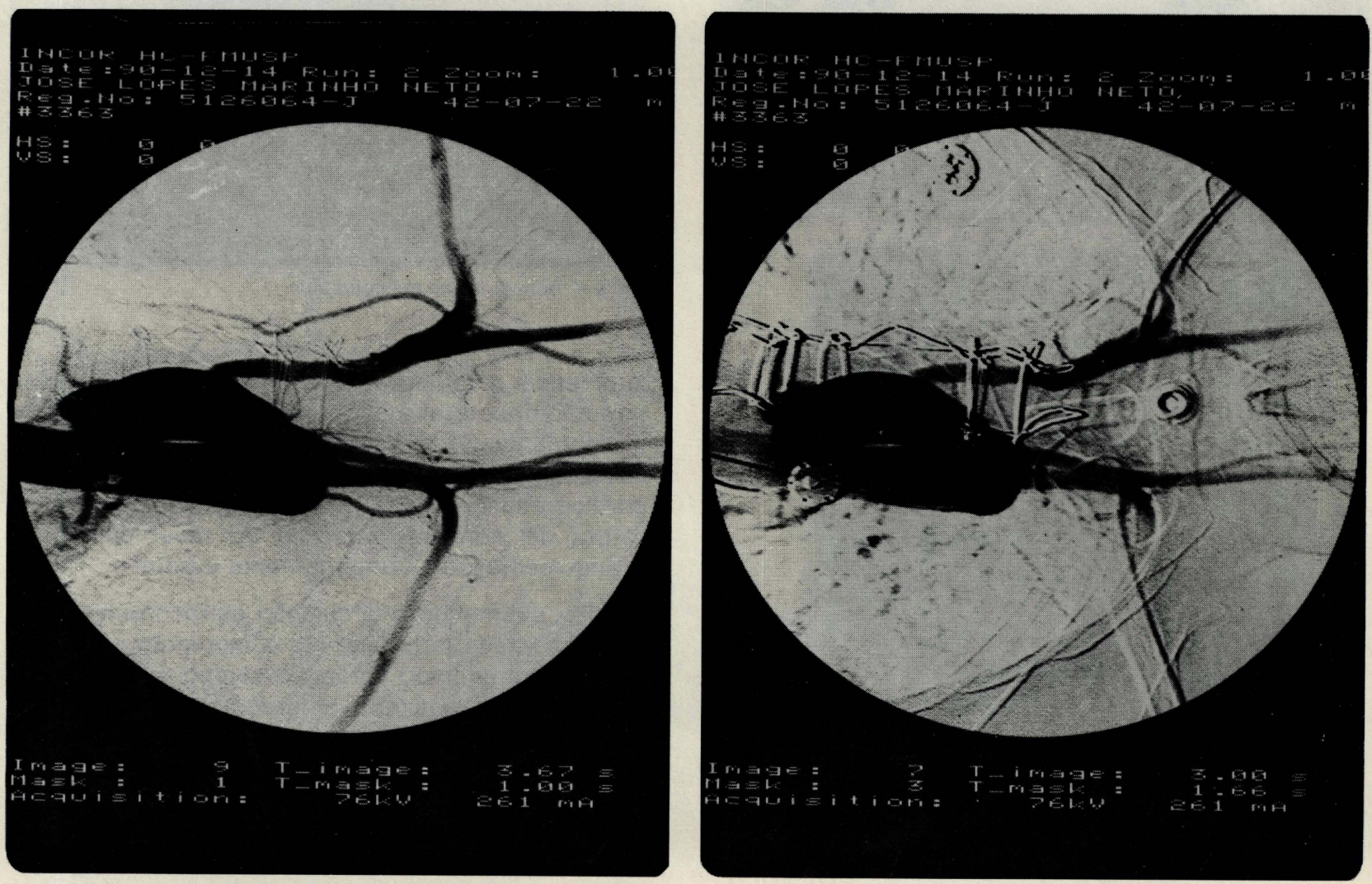

O trauma resulta, mais comumente, de lesões associadas a súbitas desacelerações em alta velocidade, seguidas de impacto, como as resultantes de acidentes com veículos motorizados, ou quedas graves 2,3. A abrupta desaceleração do corpo, como quando ele é esmagado por uma súbita parada, cria enormes forças de cisalhamento, que agem com plenitude máxima no ponto onde uma porção altamente móvel de aorta se une a um segmento fixo. Menos freqüentemente, lesōes por pressão ou explosão podem provocar rotura da aorta. Acredita-se que sejam causadas por aumento abrupto na pressão intraaórtica produzido pela compressão do sangue contido dentro da mesma e, a seguir, aumentado pela força provocada pela sístole cardíaca.

Embora a aorta possa se romper em qualquer local no seu trajeto, o ponto mais freqüente de rotura, que ocorre em cerca $90 \%$ dos casos, é o ístimo aórtico, no ponto de inserção do ligamento arterioso, junto à origem da artéria subclávia esquerda; aquí, a aorta torácica descendente torna-se fixa à parede torácica pelo ligamento arterioso, artérias intercostais e artéria subclávica esquerda.

A lesão pode variar desde um minúsculo esgarçamento na parede aórtica, até a completa transec-

Fig. 3 - Radiografia digital de controle pós-operatório. 
IGLÉZIAS, J. C. R.; PINTO, D. F.; MOREIRA, L. F. P.; DALLAN, L. A.; VERGINELLI, G.; JATENE, A. D. - Desinserção do tronco branquiocefálico em traumatismo fechado do tórax. Rev. Bras. Cir. Cardiovasc., 7(1):44-47, 1992.

ção circunferencial de todas as três camadas do vaso. Na série de PARMLEY et alii ${ }^{3}, 80 \%$ de 296 casos de trauma aórtico apresentavam rotura circunferencial.

Além das lesōes do ístmo aórtico, que é o local de
$90 \%$ de todas as lesões, áreas de lesões incluem ainda a porção supravalvar da aorta ascendente, o tronco branquiocefálico, que pode ser arrancado da aorta, arco aórtico e outros pontos da aorta torácica descendente, aorta abdominal, além da combinação de todos eles.

RBCCV 44205-161

IGLÉZIAS, J. C. R.; PINTO, D. F.; MOREIRA, L. F. P.; DALLAN, L. A.; VERGINELLI, G.; JATENE, A. D. Brachycephalic trunk rupture in closed thorax traumatism. Rev. Bras. Cir. Cardiovasc., 7(1):44-47, 1992.

ABSTRACT: The case of 48-years-old man politraumatized, victim of automobile accident is reported. The patient entered the emergency service of the Heart Institute in shock, with multiple fractures in the costal archs, total pneumothorax at the right and fracture of femur. Chest X-ray showed an important mediastinum enlargement. The patient was stabilized with fluids, monitor and submitted to angiography which revealed rupture of aorta at the level of brachycephalic trunk. Surgical treatment was undertaken, with the interposition of a Dacron tube between the ascending aorta and the brachycephalic trunk. Emphasis is given as to the way the case was approached. The approach is responsible for the good outcome reached. The procedure with the tamponage of the aortic lesion permitted suture of the rupture of the brachycephalic trunk in the aorta and the interposition of a Dacron patch between the ascending aorta and brachycephalic trunk, fully reestablishing the cerebral flux.

DESCRIPTORS: brachycephalic trunk, rupture, surgery; thorax, traumatisms.

\section{REFERÊNCIAS BIBLIOGRÁFICAS}

1 AYELLA, R. J.; HANKINS J. R.; TURNEY, S. Z.; COWLEY, R. A. - Ruptured thoracic aorta due to blunt trauma. $J$. Trauma, 17: 199-203, 1977.

2 GREENDYKE, R. M. - Traumatic rupture of the aorta: special reference to automobile accidents. JAMA, 195: 527-532, 1966.

3 PARMLEY, L. F.; MATTINGLY, T. W.; MANION, W. C.; JAHNKE, E. J. - Non penetrating traumatic injury of the aorta. Circulation, 17: 1086-1101, 1958.
4 PUIJLAERT, C. B. A. J. - Roentgen diagnosis of traumatic rupture of aorta. Radiol. Clin., 45: 217-218, 1976.

5 SPENCER, K. L.; GUERIN, P. F.; BLAKE, H. A.; BAHNSON, H. T. - A report of 15 patients with traumatic rupture of the aorta. J. Thorac. Cardiovasc. Surg., 41:1-22, 1961.

6 SYMBAS, P. N. - Great vessels injury. Am. Heart J., 93: 518-522, 1977.

7 SYMBAS, P. N.; TYRAS, D. H.; WARE, R. E.; HATACHER Jr., R. C. R. - Rupture of the aorta: a diagnostic trial. Ann. Thorac. Surg., 15:405-409, 1973. 